\title{
Characterization and test of a Data Acquisition System for PET
}

\author{
Matteo Morrocchi, Sara Marcatili, Nicola Belcari, Maria Giuseppina Bisogni, Gianmaria Collazuol, Giovanni \\ Ambrosi, Francesco Corsi, Maurizio Foresta, Cristoforo Marzocca, Gianvito Matarrese, Giancarlo Sportelli, Pedro \\ Guerra Member IEEE, Andres Santos Senior Member IEEE, Alberto Del Guerra Senior Member IEEE
}

\begin{abstract}
A small Positron Emission Tomography demonstrator based on LYSO slabs and Silicon Photomultiplier matrices is under construction at the University and INFN of Pisa.

In this paper we present the characterization results of the read-out electronics and of the detection system. Two SiPM matrices, composed by $8 \times 8 \mathrm{SiPM}$ pixels, $1.5 \mathrm{~mm}$ pitch, have been coupled one to one to a LYSO crystals array.

Custom Front-End ASICs were used to read the 64 channels of each matrix. Data from each Front-End were multiplexed and sent to a DAQ board for the digital conversion; a motherboard collects the data and communicates with a host computer through a USB port.

Specific tests were carried out on the system in order to assess its performance.
\end{abstract}

Futhermore we have measured some of the most important parameters of the system for PET application.

\section{INTRODUCTION}

$\mathrm{Sa}_{\mathrm{a}}^{\mathrm{Il}}$ ILICON Photomultipliers (SiPM) represent an effective alternative to photomultiplier tubes used in Positron Emission Tomography[1].

The reduced dimension of the SiPM and the capability to assemble array of SiPMs with a millimetric pitch allow to overcome the limit in spatial resolution imposed by the current devices.

For this reason a demonstrator of a PET module for preclinical applications is under development[2]; in small animal imaging systems, in fact, spatial resolution is a crucial parameter for the reduced anatomical dimensions of test rodens[3].

SIPMs are also attractive for PET due their insensitivity to magnetic field; therefore a new integrated PET - MRI scanner can be developed combining the functional information of

\footnotetext{
Manuscript received November 14, 2011.

Matteo Morrocchi is with University of Pisa and INFN sezione di Pisa, PI 56127 Italy (telephone: 0039-329-1262837, e-mail: matteo.morrocchi@pi.infn.it).

Sara Marcatili, Nicola Belcari, Maria Giuseppina Bisogni and Alberto Del Guerra are with University of Pisa and INFN sezione di Pisa, PI 56127 Italy. Gianmaria Collazuol is with INFN sezione di Pisa PI 56127 Italy. Giovanni Ambrosi is with INFN sezione di Perugia, PG 06100 Italy.

Francesco Corsi, Maurizio Foresta, Cristoforo Marzocca and Gianvito Matarrese are with Politecnico di Bari and INFN sezione di Bari, BA 70100 Italy.

Pedro Guerra is with CIBER-BBN, CEEI-Modulo 3, Zaragoza 50018 Spain.

Andrres Santos and Pedro Guerra are with Universidad Politecnica de Madrid, Madrid 28040 Spian, and with CIBER-BBN, Spain.
}

Positron Emission Tomography with the high anatomical resolution of a Magnetic Resonance Image technique[4].

The demonstrator presented in this paper is composed by two 8 x 8 SiPM matrices coupled to LYSO crystal matrices; a custom front - end electronics has been developed to read the devices and to communicate data to a PC.

\section{MATERIALS AND METHOD}

The detection system is composed of two SiPM matrices produced by FBK - Irst for the INFN DaSiPM2 collaboration, each SiPM is a $1,5 \mathrm{~mm} \times 1,4 \mathrm{~mm}$ and the matrix is composed by $64(8 \times 8)$ SiPMs built on a common silicon substrate, each SiPM of the matrix is composed of 840 microcelles [5]. A common bias voltage feeds the whole matrix. The two matrices are hosted on a custom board to enable the read-out of the channels. The matrix is shown in Fig. 1 (left) and the board is depicted in Fig 1 (right).
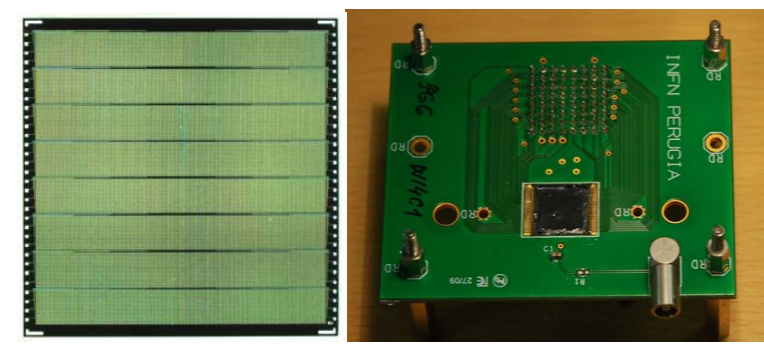

Fig. 1. SiPMs matrix used for the demonstration system: particular of the SiPMs array (left) and the custom board for the read - out (right).

Each matrix is coupled to an $8 \times 8$ LYSO crystal array, 1,5 $\mathrm{mm}$ pitch and $10 \mathrm{~mm}$ thick, so to have a one to one coupling with the SiPMs matrix; crystal and detector are coupled with optical grease in order to obtain a good coupling of the refractive indices.

The spatial resolution of the matrix has been already measured [5], coupling the device with a LYSO crystal slab and obtaining a FWHM for the distribution of the centre of mass of the event of $0,9 \mathrm{~mm}$.

The two SiPM matrices are read by a DAQ system composed of two sets of DAQ boards connected to a central motherboard[6][7], as shown in Fig. 2.

Each DAQ board hosts a custom front-end ASIC [8] developed by the INFN of Bari - Politecnico of Bari. The chip has 8 input channels, so 8 DAQ boards are needed to read the whole SiPMs matrix. Each channel of the ASIC is composed 
of a Current Buffer with a $250 \mathrm{MHz}$ bandwidth and input impedance lower than $20 \mathrm{Ohm}$. The signal coming from the current buffer is splitted in two paths. One is connected to a Current Discriminator; the trigger signal generated by the ASIC is obtained by a logic OR between the signal of the eight discriminators of the channels. The threshold level could be set at 12 different levels using four configuration bits. The other branch is connected to a CSA (Charge Sensitive Amplifier) to integrate the signal with three different gain levels and then to a Peak Detector to keep the signal fixed to a costant level during the reading process. The integration time of the CSA is set to $250 \mathrm{~ns}$ according to the decay time of the scintillator crystal.

The DAQ hosts a 10 bits ADC and an FPGA Altera Cyclone II used to communicates with the motherboard and the ASIC.

The central motherboard hosts a FPGA Altera Stratix III used to manage the $\mathrm{I} / \mathrm{O}$ signals to/from the DAQ and to perform the time coincidence, data are stored in a FIFO and communicated to a host PC using a USB protocol.

At the moment two DAQ boards are completed and available and others are under construction.

In Fig. 3 the motherboard and the two DAQ boards used in the work are represented.

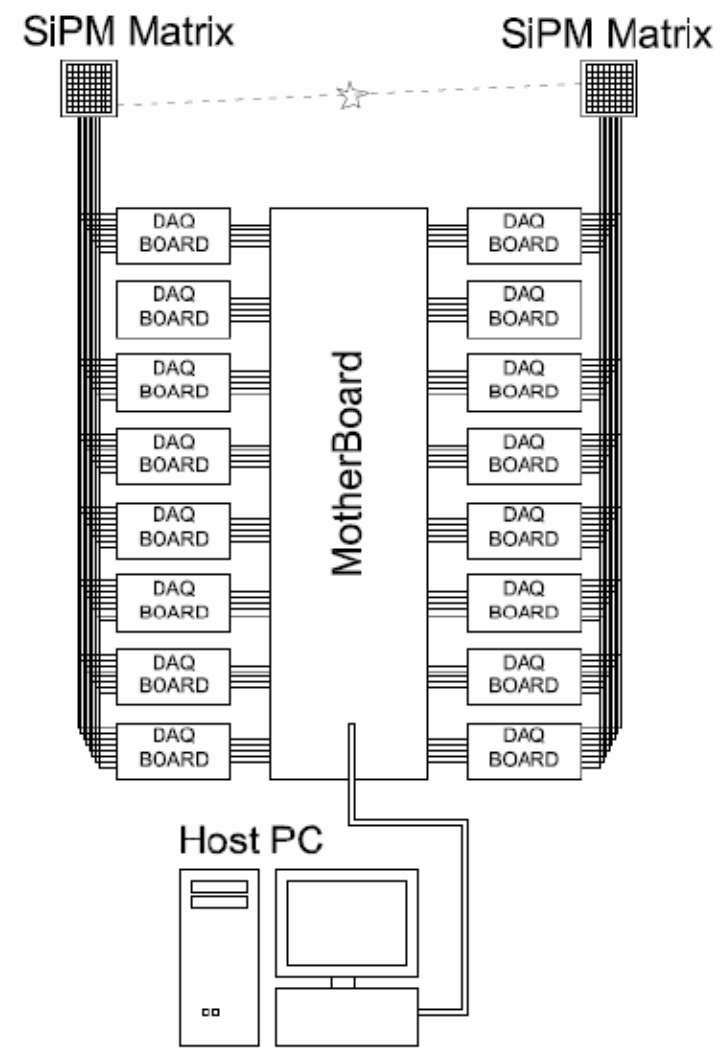

Fig. 2. Scheme of the detection system.

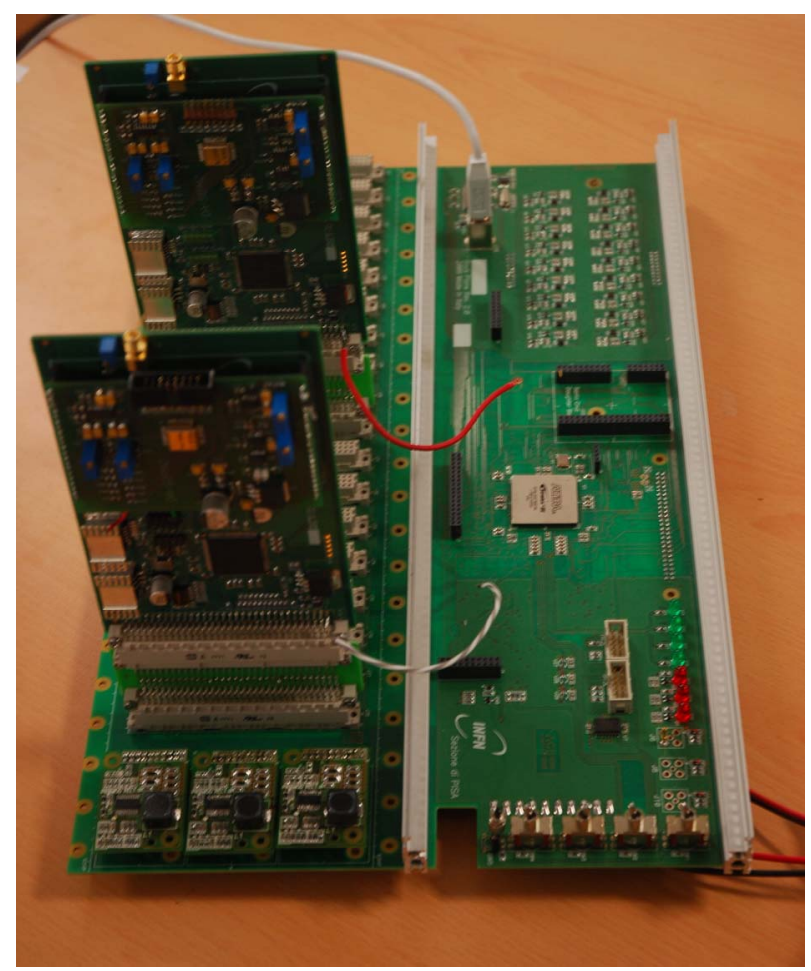

Fig. 3. The acquisition system composed on a central motherboard and two DAQ boards.

\section{READ - OUT SYSTEM CHARACTERIZATION}

The first measurements have been executed to characterize the performance of the two Front - End ASICs. The linearity has been tested for each channel of both devices, injecting different values of charge to simulate the signal generated by the LYSO - SiPM module.

A shaping circuit connected to a waveform generator has been used to reproduce the tipical signal shape obtained with crystal and SiPM. The mean value of the signal distribution has been considered for each amplitude of the charge injection.

The plots of the ADC channel as a function of amplitude of the square wave for the two chips are shown in Fig. 4.

The value of the pedestal for each channel has been obtained using a linear fit.

The threshold values have been quantified by the same test signals. A defined number of pulses has been sent to the ASIC and the number of triggered events has been plotted as a function of the amplitude of the current peak of the signals. The error function so obtained has been derivated and the threshold level has been obtained with a gaussian fit.

The Error Function plot of the lower threshold level is shown in Fig. 5, and the result of the first 8 levels is summarized in Fig. 6; as can be seen, the spacing between levels is constant. 


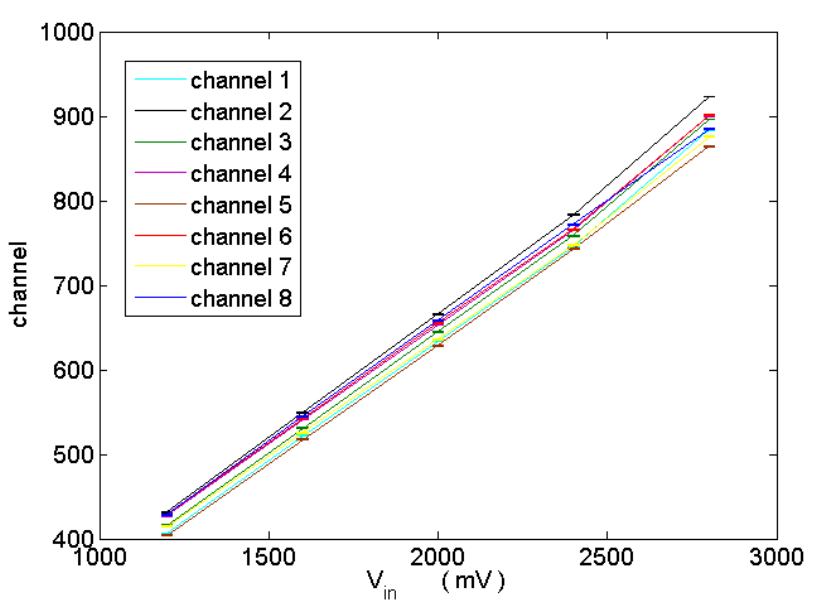

(a)

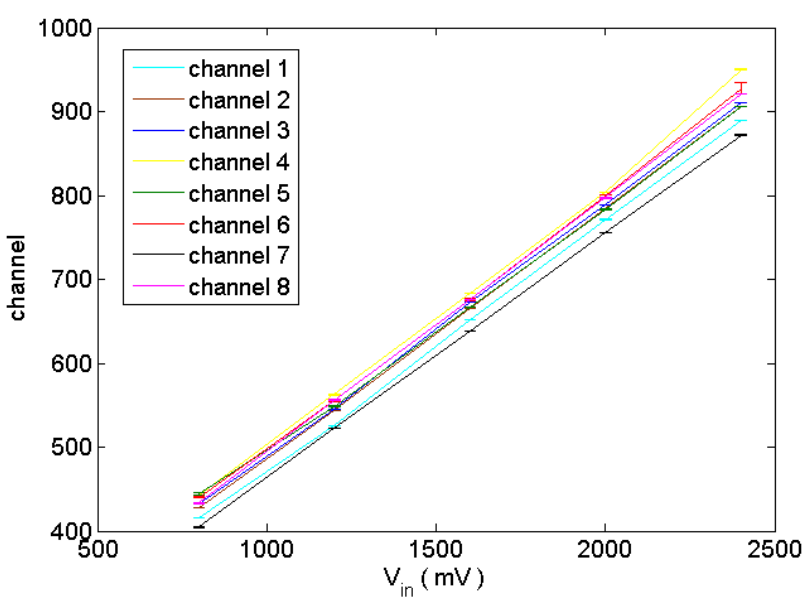

(b)

Fig. 4. Linearity test for the 8 channels of the first (a) and second (b) ASIC. The average ADC channel is plotted as a function of the amplitude of the signal of the pulse generator.

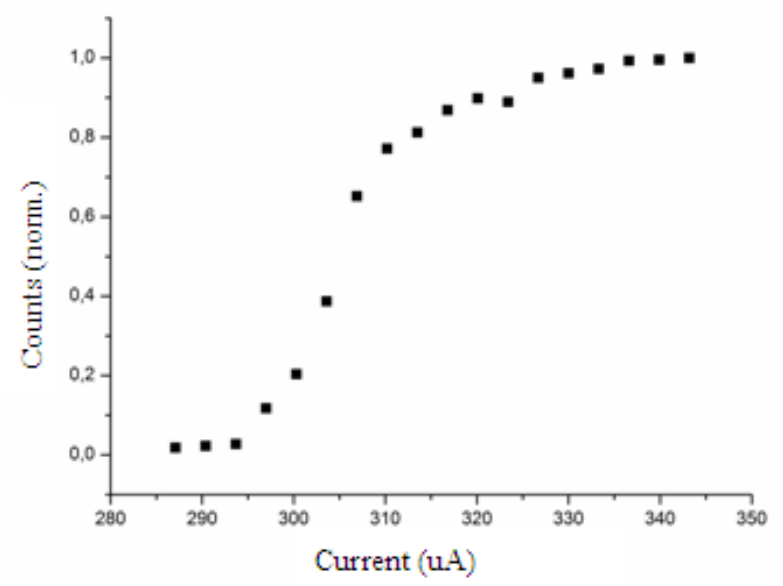

Fig. 5. Error function obtained with the lowest threshold level.

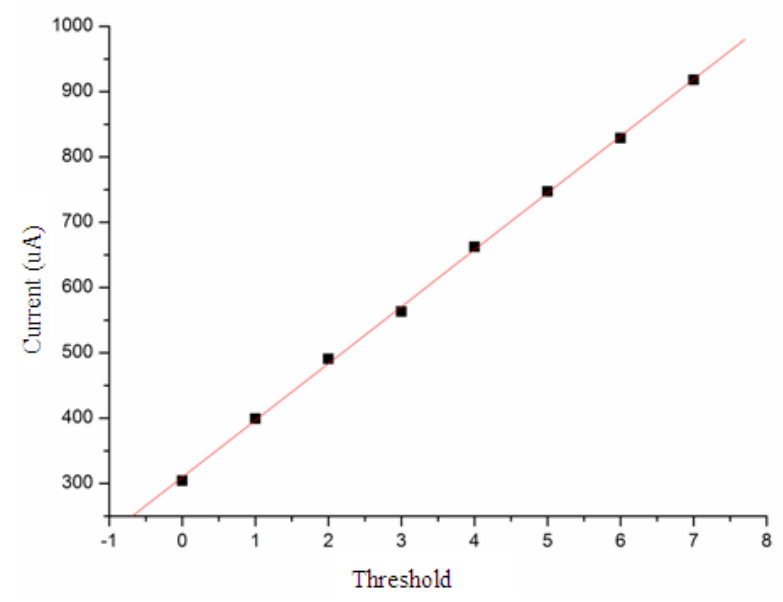

Fig. 6. The current peak injected in the ASIC is plotted as a function of the level of threshold.

Timing perrformances has been finally assessed by sending test signals in time coincidence to the two ASICs.

The two digital trigger signals generated by the Current Discriminator have been sent to an oscilloscope with passband of $1,5 \mathrm{GHz}$ and sampling rate of $2 \mathrm{GS} / \mathrm{s}$.

The time jitter $\sigma_{\mathrm{t}}$ depends on the amplitude of the signals and on the noise level of the system according to the equation[9]:

$$
\sigma_{t}=\frac{\sigma_{n}}{(d S / d t)} \approx \frac{t_{r}}{S / N}
$$

In which $\sigma_{\mathrm{n}}$ is the standard deviation of the noise, $t_{r}$ is the rise - time of the signal, $S$ is its amplitude and $N$ is the noise.

The distribution of the time difference between the two signals is plotted and fitted obtaining as best result a FWHM of $160 \pm 6$ ps, as shown in Fig. 7 .

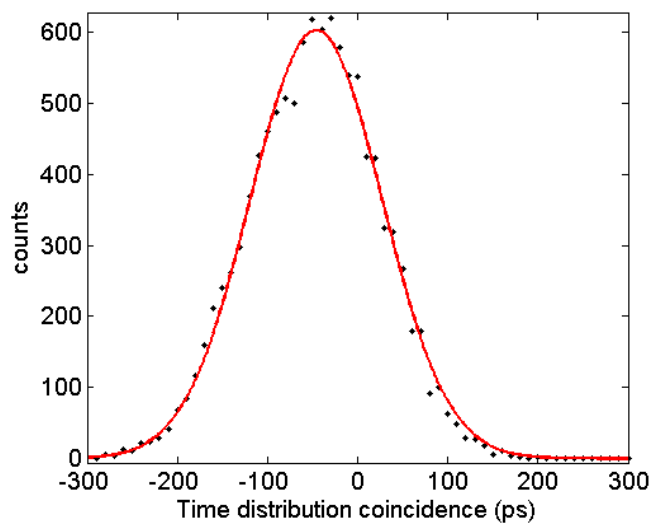

Fig. 7. Time delay distribution between the trigger signals of the two ASICs using test signals.

\section{DETECTION SYSTEM CHARACTERIZATION}

In order to measure the performances of the whole detection system (comprehending crystal, SiPMs and read - out electronics) the two ASICs have been connected to a line of the SiPM's matrix (8 pixels).

Timing measurements have been performed using a ${ }^{22} \mathrm{Na}$ source between the two lines of detectors and the lowest threshold. Due to the leading edge discrimination method used 
in the chip, the system time resolution is limited by time walk effect of signals of same rise time and different amplitude. To avoid the saturation of the CSA an appropriate bias voltage for the two matrices has been chosen.

The distribution of the time delay of the two triggers is shown in Fig. 8 and it shows a FWHM of 1,8 $\pm 0,2$ ns.

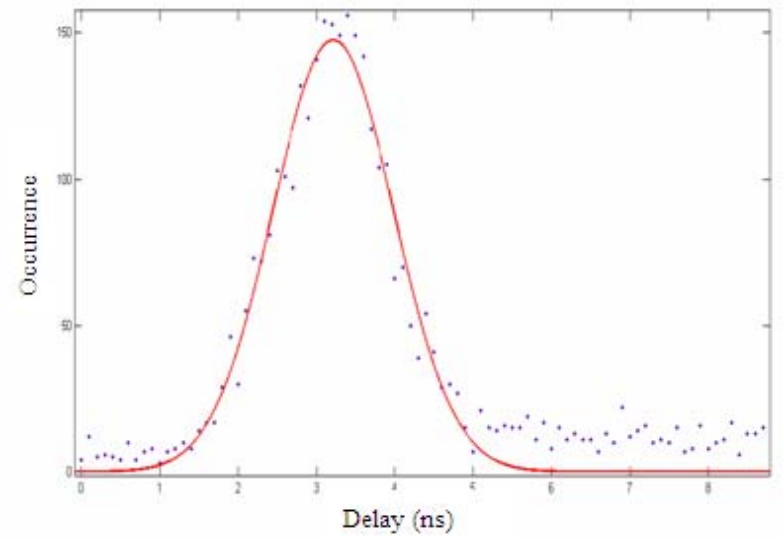

Fig. 8. Time delay distribution between the trigger signals of the two ASICs using. ${ }^{22}$ Na souces.

Energy resolution has been estimated using both single line and two lines in time coincidence; the value of the pedestals previously founded have been used to calibrate the channels.

Using a single line, the FWHM of the $511 \mathrm{keV}$ peak of the ${ }^{22} \mathrm{Na}$ results in $15,3 \pm 0,4 \%$ and using the two devices in coincidence the result is $16,9 \pm 0,4 \%$.

The spectrum of single events is shown in Fig. 9.

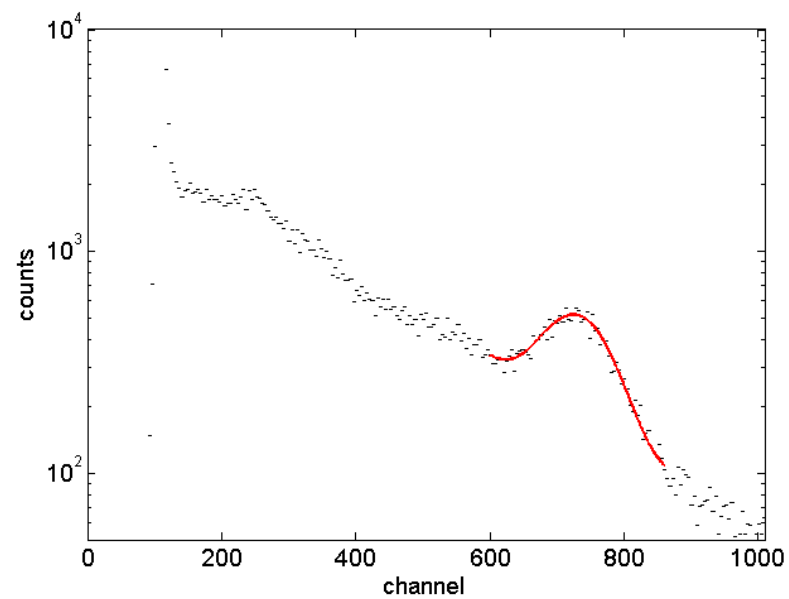

Fig. 9. ${ }^{22} \mathrm{Na}$ spectrum acquired with a single line of 8 SiPMs with a 1 to 1 coupling to LYSO crystal of $10 \mathrm{~mm}$ width.

To evaluate the imaging capabilities of the system, the two SiPM matrices has been set to $120 \mathrm{~mm}$ distance and the souce has been moved in 5 different positions in the transversal direction. A coincidence window of $10 \mathrm{~ns}$ has been chosen. The set - up is shown in Fig. 10. Variating the distance of the source from the axis of the two matrices inside the Field of View (FOV) of the two lines, the position has been reconstructed taking into account the occurrence in each Line of Response (LOR).

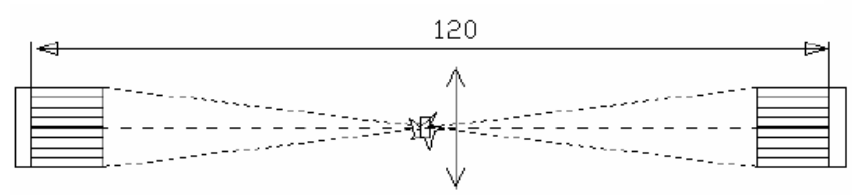

Fig. 10. Set-up for the test of position reconstruction with the matrices at a distance of $120 \mathrm{~mm}$.

As could be seen in Fig. 11 the position could be well reconstructed in all the situations analyzed; the error in the y axis is determined by the FWHM of the distribution of the LOR in the tansversal direction while the $\mathrm{x}$ error depends on the positioning of the source.

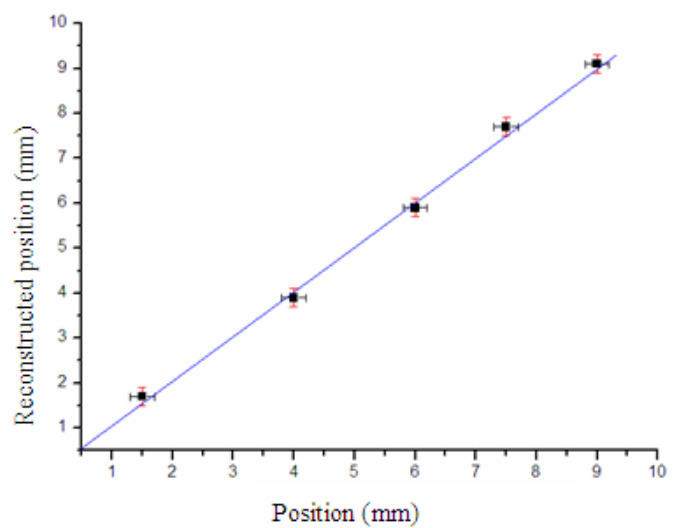

Fig. 11. Reconstructed position as a function of the real position of the source with the two natrices at $120 \mathrm{~mm}$ distance. The centre of the FOV is at the midline.

\section{CONCLUSIONS}

A demonstrator for preclinical Positron Emission Tomography application composed of two SiPMs matrices and LYSO crystal is under development and the first results for the performances of the detection system are presented.

The ASIC performances are complient with PET requirements; the electronic time jitter has been quantified to $160 \pm 6$ ps (FWHM).

Two DAQ boards have been used to read one line for each SiPM matrix. An energy resolution for the $511 \mathrm{keV}$ peak of ${ }^{22} \mathrm{Na}$ of $15,3 \pm 0,4 \%$ has been obtained using a single line and a result of $16,9 \pm 0,4 \%$ has been obtained with the two lines in coincidence.

A time resolution of $1,8 \pm 0,2 \mathrm{~ns}$ (FWHM) has been measured using a single discrimination method. This result is affected by the time walk because photopeak events can not be selected with the present set-up; a better performance is expected with a double threshold composed of a low level for time information and a higher level at the peak energy.

\section{REFERENCES}

[1] W. W. Moses, Photodetectors for nuclear medical imaging, Nuclear Instruments and Methods in Phisics Research A 610 (2009) 11-15.

[2] S. Marcatili et al., Development and characterization of a modular acquisition system for a 4D PET block detector, Nuclear Inst. and Methods in Physics Research, A 659 (2011), pp. 494-498

[3] A. Del Guerra, N. Belcari, State-of-the-art of PET, SPECT and CT for small animal imaging, Nuclear Instruments and Methods in Physic A 583 (2007) 119-124. 
[4] N. Ohja et al.,m PET performance of the GEMINI TF PET - MR: The world's first whole body PET - MRI scanner, Nuclear Science Symposium Conference Record (NSS/MIC), 2010 IEEE, DOI:10.1109/NSSMIC.2010.5874129

[5] Llosa et al., Characterization of a PET detector head based on continuous LYSO crystal and monolytic 64-pixel silicon photomultiplier matrices. 2010 Phis Med Biol 557299.

[6] G. Sportelli et al., Reprogrammable Acquisition Architecture for Dedicated Positron Emission Tomography, IEEE Transaction on nuclear science 2011, vol. 3 pp. 695-702.

[7] G. Sportelli et al., A flexible acquisition system for modular dual head Positron Emission Mammography, Nuclear Science Symposium Conference Record (NSS/MIC), 2009 IEEE, pp.3395 - 3398.

[8] F. Corsi et al., N19-2, BASIC: an 8-channel Front-end ASIC for Silicon Photomultiplier Detectors 2009 IEEE Nuclear Science Symposium Conference Record 2009, pp. 1082-1087.

[9] H. Spieler, Fast Timing Methods for Semiconductor Detectors, IEEE Trans. Nucl. Sci. NS-29/3 1142- 1158. 